\title{
THE LAPLACE HEAVISIDE METHOD FOR BOUNDARY VALUE PROBLEMS*
}

\author{
D. G. BOURGIN AND R. DUFFIN
}

The usual methods for solution of boundary value problems for linear differential equations are based on taking linear combinations with "undetermined" coefficients of particular solutions of the homogeneous or nonhomogeneous equation. Subsidiary calculations are then required to deduce coefficient values such that the boundary conditions are satisfied. This note is concerned primarily with the formulation of a novel viewpoint in the Laplace integral treatment of differential equations and presents two new methods, neither involving undetermined coefficients, which are alternatives to the standard procedures.

Our developments stem from relations $(\alpha)$. The Laplace transform $F(p)$ plays the determining role. This function does not appear in the current developments of the definite integral solutions for variable coefficient equations. Since our methods yield solutions automatically satisfying prescribed boundary relations, they bear the same relationship to other procedures as does the Heaviside method, heretofore available for the one point problem for constant coefficient equations. Indeed our Method 1 is shown to be the natural generalization of the Heaviside procedure.

Consider, for real $a, b$,

$$
F(p)=\int_{a}^{b} y(x) e^{-x p} d x, \quad-\infty<a<b<\infty .
$$

Then, for $y(x)$ continuous,

$(\alpha 1) F(p)$ is entire and of exponential type,

$(\alpha 2)|F(p)| \leqq M\left(e^{-a \sigma}+e^{-b \sigma}\right) /|\sigma|, \sigma=\mathbb{R}(p) . \dagger$

Furthermore

$$
y(x)=1 / 2 \pi i \int_{c-i \infty}^{c+i \infty} e^{x p} F(p) d p, \quad c \text { a real positive constant. }
$$

For much of our work we shall be interested in the system $\ddagger$

* Presented to the Society, November 28, 1936, under the title $A$ method for certain two-point boundary value problems.

$\dagger$ Extensions involving the Riemann-Lebesgue lemma or differentiability properties of $y(x)$ are unnecessary for our present purpose.

$\ddagger$ The differential equation terminology in this article is consistent with that of Ince, Ordinary Differential Equations. This book is designated by I in subsequent references. 


$$
\begin{aligned}
L(x, d / d x) y & \equiv \sum_{j=0}^{n} a_{j}(x) d^{j} / d x^{j} y(x)=r(x), \\
U_{i}(a, b) & \equiv \sum_{j=0}^{n-1} \alpha_{i j} y^{j}(a)+\beta_{i j} y^{j}(b)=d_{i}, \quad i=1, \cdots, n,
\end{aligned}
$$

where $a_{i}(x)$ is linear, $\alpha_{i j}, \beta_{i j}, d_{i}$ are constants and (2) is of rank 1.* In the sequel, bars over operators will denote adjoints and $P()$ the bilinear concomitant. Furthermore, we assume: (A) $a_{n}(x) \neq 0$ for $a \leqq x \leqq b,(\mathrm{~B}) r(x)$ is piecewise continuous, (C) the rectangular matrix $\left\|\alpha_{i j}, \beta_{i j}\right\|$ is of rank $n$. We make a customary restriction: (D) to solutions continuous together with their first $n-1$ derivatives for $-\infty<a \leqq x \leqq b<\infty$. Restrictions (A), ..., (D) guarantee, also, piecewise continuity of the first derivative (that is, $y^{\prime}(x)$ ) which is sufficient here for the ordinary convergence of the right-hand integral in (1.1) to $y(x)$. The relation $y(x) \doteq F(p)$ connotes the simultaneous validity of (1) and (1.1). $\dagger$

The constant coefficient case already brings out the utility of relations $(\alpha)$. Here

$$
\begin{aligned}
& L(d / d x) . \equiv \sum_{0}^{n} a_{j} d^{j} / d x^{j} ., \quad L(d / d x) .=L(-d / d x) . \\
& \int_{a}^{b}\left\{e^{-x p} L(d / d x) y(x)-y(x) \bar{L}(d / d x) e^{-x p}\right\} d x \\
&=\left.P\left(y^{i}(x), p, e^{-x p}\right)\right|_{a} ^{b} .
\end{aligned}
$$

Therefore

$$
F(p) L(p)=R(p)
$$

where

$$
R(p)=\int_{a}^{b} e^{-x p} r(x) d x-\left.P\left(y^{i}(x), p, e^{-x p}\right)\right|_{a} ^{b} .
$$

Let the $J$ distinct roots $\left\{p_{i}\right\}$ of $L(p)$ be of multiplicities $\left\{m_{i}\right\}$; then $\sum_{i=1}^{J} m_{i}=n$. The root $p_{j}$ must be a zero of $R(p)$ of order $m_{j}$ at least, since $F(p)$ is entire. Thus the following $n$ equations are satisfied:

* I, p. 427.

$\dagger$ For the notation and the facts used here concerning the Laplace-Mellin representation of the Heaviside calculus, cf. for instance D. G. Bourgin and R. J. Duffin, American Journal of Mathematics, vol. 59 (1937), p. 489. (Cf. G. Doetsch, Theorie und Anwendung der Laplace Transformation, Berlin, 1937, and the papers of D. V. Widder for further properties.) 


$$
d^{\sigma} /\left.d p^{\sigma} R(p)\right|_{p=p_{j}}=0, \quad \sigma=0, \cdots, m_{j}-1 ; j=1, \cdots, J,
$$

with the convention $d^{0} / d p^{0} R(p)=R(p)$.

The $2 n$ equations, (2.1) and (2.4), are linear in the unknowns $y(a), \cdots, y^{(n-1)}(a), y(b), \cdots, y^{(n-1)}(b)$. If the coefficient and augmented matrices are of rank $2 n-k$, the general solutions for these unknowns, in terms of $\alpha_{i j}, \beta_{i j}, d_{i}, a_{i}, p_{j}$ and $\int_{a}^{b} x^{\sigma} e^{-p_{j} x} y(x) d x$, involve $k$ essential parameters.

The solution required is therefore

$$
y(x) \doteq[R(p)] / L(p)(\equiv F(p)) .
$$

The brackets denote here, and in (2.51), that the $y(a), \cdots, y^{n-1}(b)$ occurring in $R(p)$ are replaced by their determinate values (as found here from (2.1) and (2.4)). This method of solution is a special case of Method 1, described below.

We now take up in detail the case of linear coefficients. The analysis may be extended in an obvious manner to the case of polynomial coefficients for the differential equation of rank 1 . We have

$$
\begin{aligned}
L(x, d / d x) y(x) & \equiv L_{0}(d / d x) y(x)+x L_{1}(d / d x) y(x) \\
& \equiv \sum_{j=0}^{n}\left(a_{j}+x b_{j}\right) d^{j} / d x^{j} y(x)=r(x),
\end{aligned}
$$

with $b_{n} \neq 0$. Thus

$$
\begin{gathered}
L(x, d / d x) .=L_{0}(-d / d x) .+L_{1}(-d / d x) x . \\
\int_{a}^{b}\left\{e^{-x p} L(x, d / d x) y(x)-y(x) \bar{L}(x, d / d x) e^{-x p}\right\} d x \\
=\left.P\left(x, y^{i}(x), p, e^{-x p}\right)\right|_{a} ^{b}
\end{gathered}
$$

that is,

$$
\int_{a}^{b} y(x) \bar{L}(x, d / d x) e^{-x p} d x=\int_{a}^{b} e^{-x p} r(x) d x-\left.P()\right|_{a} ^{b} \equiv R(p) .
$$

A straightforward reduction of the left side of (3.21) yields

$$
M(p, d / d p) F(p) \equiv L_{0}(p) F(p)-\frac{d}{d p}\left(L_{1}(p) F(p)\right)=R(p) .
$$

Let $G(p)$ satisfy

$$
\bar{M}(p, d / d p) G(p) \equiv\left\{L_{0}(p)+L_{1}(p) d / d p\right\} G(p)=0,
$$


that is,

$$
G(p)=\exp \left[-\int^{p} L_{0}(p) / L_{1}(p) d p\right]
$$

Then

$$
\int_{\gamma}\{G(p) M(p, d / d p) F(p)-F(p) \bar{M}(p, d / d p) G(p)\} d p=\left.G(p) F(p) L_{\mathbf{1}}(p)\right|_{\gamma},
$$

or

$$
\int_{\gamma} G(p) R(p) d p=\left.G(p) F(p) L_{1}(p)\right|_{\gamma}
$$

where $\gamma$ is to be chosen so that $\left.F(p) G(p) L_{1}(p)\right|_{\gamma}$ vanishes.

Suppose the roots of $L_{1}(p)$ are $\left\{p_{i}\right\}, i=1, \cdots, l$, where $p_{i}$ is of multiplicity $m_{i}>1$ for $i=1, \cdots, r$ and is a simple root for $r<i \leqq l$. We may write (3.41) in the form

$G(p)=\exp \left[s p+\sum_{i=1}^{r} \sum_{j=1}^{m_{i}-1} B_{i j} /\left(p-p_{i}\right)^{j}\right]\left(p-p_{1}\right)^{A_{1}} \cdots\left(p-p_{l}\right)^{A_{l}}$,

where $s=-a_{n} / b_{n}$. In view of (A) we may assume $s<a$ (though it may be necessary to multiply both sides of (3.1) by -1 to achieve this situation). The $\left\{A_{k}\right\}$ and $\left\{B_{i j}\right\}$ are constants, some or all of which may be 0 when $L_{0}(p)$ and $L_{1}(p)$ have roots in common.

The paths $\left\{\gamma^{\prime}\right\}$ obtained by the usual application of the Laplace transformation to the homogeneous equation associated with (3.1) are the same as those obtained from its adjoint and satisfy*

$$
\left.e^{-x p} G(p) L_{1}(p)\right|_{\gamma^{\prime}}=0 .
$$

So far as (3.5) is concerned, $F(p)$ need be considered solely in connection with approach to $\infty$ and indeed (cf. $(\alpha)$ ) may be replaced by $e^{-a \sigma}$ (thus justifying termination of the path at $p=\infty$ ). (The factor $|\sigma|^{-1}$ is unimportant since $s \neq a, b$. Cf. (A).) Hence, on identifying $x$ in (3.51) with $a$, it is plain that the of ten catalogued available paths $\dagger\left\{\gamma^{\prime}\right\}$ and $\{\gamma\}$ are the same when restriction is made to the case $|J(p)|<\infty$ for points on a path.

The function $R(p)$ of (3.21) may be written

(4) $\left.e^{-x p} \sum_{j=0}^{n-1} y^{(j)}(x)\left\{L_{0}^{j+1}(p)+(x-d / d p) L_{1}^{j+1}(p)\right\}\right|_{a} ^{b}-\int_{a}^{b} e^{-x p} r(x) d x$

* I, p. 438.

$\dagger \mathrm{I}, \mathrm{pp} .455,457,459$. 
where

$$
p^{j} L_{\alpha}^{j}(p)
$$$$
\alpha=0,1,
$$

represents the remainder of $L_{\alpha}(p)$ after the $j$ terms of lowest degree are removed. From (3.5) we have

$$
\begin{aligned}
y(b) F_{l 0}(b)+\cdots+y^{(n-1)}(b) F_{l n-1}(b) & -y(a) F_{l 0}(a)-\cdots \\
& -y^{(n-1)}(a) F_{l n-1}(a)=\int_{\gamma l} G(p) \int_{a}^{b} e^{-x p} r(x) d x d p,{ }^{*}
\end{aligned}
$$

where

$$
\begin{array}{r}
F_{l j}(z)=\int_{\gamma} e^{-z p} G(p)\left\{L_{0}^{j+1}(p)+z L_{1}^{j+1}(p)-d / d p L_{1}^{j+1}(p)\right\} d p, \\
l=1, \cdots, n .
\end{array}
$$

Relation (6) is central in our work. For applications we need

$$
\left|\cdot F_{l j}(x):\right| \neq 0, \quad a \leqq x \leqq b .
$$

We proceed now to establish relation (6.1). We introduce the notation (superscripts in parentheses indicate differentiation here and in the sequel)

$$
\begin{aligned}
& V_{l}(x)=\int_{\gamma_{l}} e^{-x p} G(p) d p,
\end{aligned}
$$

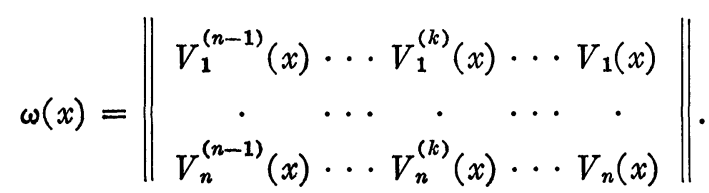

The determinant of this matrix is the Wronskian of $\left\{V_{l}(x)\right\}$ and is denoted by $W(x)$. It may be shown directly that

$$
\begin{aligned}
\int_{\gamma_{l}} e^{-x p} p^{j} G(p) d p & =(-1)^{j} d^{j} / d x^{j} V_{l}(x), \\
\bar{L}(x, d / d x) V_{l}(x) & =0,
\end{aligned}
$$

$$
\left(a_{n}+x b_{n}\right)(-1)^{j} V_{l}^{(j)}(x)=\sum_{\sigma=0}^{j-1} k_{\sigma}(x) F_{l \sigma}(x)+F_{l j}(x)
$$

* The constants appearing are $y(a), \cdots, y^{n-1}(b)$ and not "undetermined" coefficients as in the usual methods. Thus, whenever, as in the one point problem, the value of $y(a)$ and the $n-1$ derivatives are given explicitly, the solution may be written down at once without the necessity of calculation of the values of the "undetermined" or arbitrary constants required in the non-Heaviside type procedures. 
where $\left|k_{q}(x)\right|<\infty, q=0, \cdots, n-1$, for $x>s$. From (6.5) we derive

$$
\left|\cdot F_{l j}(x):\right|=\left(-a_{n}-x b_{n}\right)^{n} W(x)
$$

For large $x, n$ contours $\left\{\gamma_{l}\right\}$ and solutions $\left\{V_{l}(x)\right\}$ of (6.5) always exist for which $W(x) \neq 0$.* Hence $W(x) \neq 0$ for $a \leqq x \leqq b$ by virtue of the Abel identity. $\dagger$ Since $s<a$, the verification of (6) is complete.

Method 1. Equations (2.1) and (6) provide a linear simultaneous system in the unknowns $y(a), \cdots, y^{(n-1)}(a), \cdots, y^{(n-1)}(b)$. By (6.1) and (6.6) the coefficient determinant may be written in the form

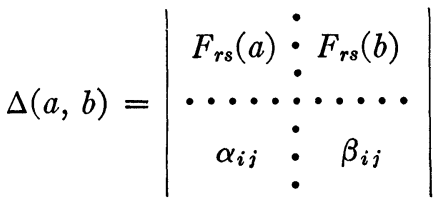

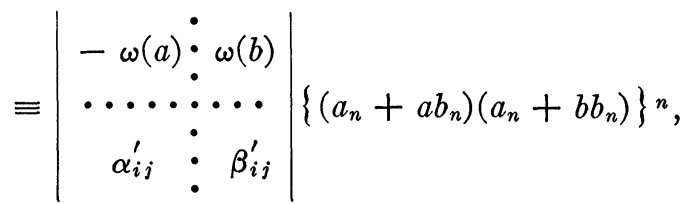

where (cf. (6.5))

$$
\alpha_{i j}^{\prime}=\alpha_{i j}+\sum_{\sigma=0}^{j-1} k_{\sigma}(a) \alpha_{i \sigma}, \quad \beta_{i j}^{\prime}=\beta_{i j}+\sum_{\sigma=0}^{j-1} k_{\sigma}(b) \beta_{i \sigma} .
$$

For purposes of exposition we make the hypothesis (E): $\Delta(a, b) \neq 0$. We may therefore solve for the unknowns mentioned and substitute their values in $R(p)$, thus obtaining $[R(p)]$.

In view of (3.21) and (3.5)

$$
G(p) F(p) L_{1}(p)=C+\int^{p} G(p)[R(p)] d p .
$$

The left side of (7.1) vanishes under our conditions at $p=\infty$. Hence, compare (2.5),

$$
y(x) \doteq F(p)=\int_{\infty}^{p} G(p)[R(p)] d p / G(p) L_{1}(p) .
$$

The integration contour is a simple curve avoiding the singularities

* Cf. I, references, pp. 444,445 .

$\dagger$ I, p. 119. 
$\left\{p_{j}\right\}$ of $G(p)$ and branch cuts.* However, no difficulty over other branches of $F(p)$ can arise since, by $(\alpha), F(p)$ is single valued.

Method 1 may be viewed fairly as a generalization of the Heaviside procedure. We outline the argument. We restrict attention to the case that condition (A) is satisfied, that $r(x) \subset L(a, \infty)$ and is continuous for $x \geqq a$, that $\left\{p_{j}\right\}$ are distinct roots of $L(p)$ for (2) and of $L_{1}(p)$ for $(3.1)$, and that $R\left(p_{j}\right)<0 \leqq a$.

Under these restrictions it is easily shown that $|y(x)|<M$ for $x \geqq a . \dagger$ The function $y^{(i)}(x)$ appears in $P()$ only as part of a product $e^{-x p} y^{(i)}(x) q_{i}(x)$, where $q_{i}(x)$ is a polynomial. Accordingly

$$
L_{b \rightarrow \infty} P\left(y^{i}(b), p, e^{-b p}\right)=0 .
$$

Since $y(a), \cdots, y^{(n-1)}(a)$ are known here and (7.2) justifies omission of $y(b), \cdots, y^{(n-1)}(b)$ for $b \rightarrow \infty$, we can write down $[R(p)]$ in (2.5) or (2.51) immediately without the mediation of the contours $\left\{\gamma_{l}\right\}$. (Evidently the Laplace integral in (1) exists for $R(p)>0$ with $b$ replaced by $\infty$, in virtue of the finiteness of $y(x)$; and, in fact, $y(x) \doteq F(p)$, since $y^{(1)}(x)$ is easily shown to be continuous for $x \geqq a$.) In the constant coefficient equation, the result is identical with the Bromwich or Mellin integral formuation of the classic Heaviside solution, extended to nonzero data, for the one point problem.

Method 2. An alternative solution of the two-point problem is available. On introducing the intermediate point $X$ in (6) we may write

$$
\left.\sum_{k=0}^{n-1} y^{(k)}(x) F_{l k}(x)\right|_{a} ^{X}=\int_{\gamma_{l}} \int_{a}^{X} e^{-x p} r(x) d x, \quad l=1, \cdots, n .
$$

Relations (6) and (8), together with (2.1), provide $3 n$ equations in the $3 n$ unknowns, $y(a), \cdots, y^{(n-1)}(a), y(b), \cdots, y^{(n-1)}(b)$, $y(X), \cdots, y^{(n-1)}(X)$. The denominator determinant in the solution for $y(X)$ by Cramer's rule is reducible to $\left(-a_{n}-b_{n} X\right)^{n} \cdot W(X) \cdot \Delta(a, b)$. Under hypothesis $(\mathrm{E})$ the unique solution $y(X)$ exists, satisfying the system (3.1) and (2.1).

Method 2 also yields a solution of the one point problem. Indeed if $\beta_{i j}=0$ in (2.1), we solve for $y(X)$ from (2.1) and (8). The denominator $\left|F_{l j}(X)\right|\left|\alpha_{i j}\right|$ and is nonzero for $X \geqq a$ by (C) and (6.1).

If $r(x)=0$ and a parameter $\lambda$ enters linearly in (2) or (3.1) or in (2.1), we have the characteristic function problem. The procedure for

* The particular choice of $\left\{p_{n_{i}}\right\}$ with $A_{n_{i}}>-1$ (where the direction of approach to $p_{n_{i}}$ is determined by the signs of the associated $B_{n_{i j}}$ if $B_{n_{i j}} \neq 0$ ), or of $\infty$ as the lower limit in (2.51) is of no consequence, since, for the case $A_{n_{i}}>-1$, the $\gamma i^{\prime} \mathrm{s}$, determined earlier, include the contours joining $p_{n_{i}}$ and $p_{n_{j}}$ or $p_{n_{i}}$ and $\infty$ so that $\int_{p_{n_{j}, \infty}, p_{i}}^{\infty} G(p)[R(p)] d p=0$.

$\dagger$ I, p. 441, paragraph 18.12 . 
solution amounts to determining such values for $\lambda$ that the algebraic systems (2.1) and (2.4) or (2.1) and (6) have a nonzero solution. This amounts to finding $\lambda$ values for which $[R(p)]=0$. Methods 1 and 2 then apply. Since the analysis is straightforward enough we take up instead an instance of the sort of characteristic function problem arising in modern quantum theory, namely

$$
\begin{aligned}
& L(x, d / d x) y \equiv x y^{(2)}(x)+\left(2-x k^{2}\right) y(x)=0, \\
& y(x), y^{(1)}(x) \subset L(0, \infty) ; \Im(k)=0 .
\end{aligned}
$$

We note that (9) guarantees the continuity of $y(x), y^{(1)}(x)$ and $y^{(2)}(x)$ for $x>a$. Equation (9.1) implies

$$
L_{x \rightarrow 0} x y(x)=0 ; \quad L_{x \rightarrow 0} x y^{(1)}(x)=0 ; k^{2}>0 .
$$

Moreover the Laplace integrals of $y, y^{(1)}, x y^{(1)}$ certainly exist for $R(p)>0$. Equation (9) is then the basis for the assertion that the Laplace integral of $x y^{(2)}$ exists for $R(p)>0$. The analogues of relations $(\alpha)$ are (a) $F(p)$ is analytic for $R(p)>0$; (b) $L R(p) \rightarrow \infty p F(p)=0$. Now

$$
\begin{aligned}
\int_{\bar{x}}^{\infty}\left\{e^{-x p} L(x, d / d x) y-y \bar{L}(x, d / d x) e^{-x p}\right\} d x & \\
& =\left.\left[e^{-x p}\left(y(x p-1)+x y^{\prime}\right)\right]\right|_{\bar{x}} ^{\infty} .
\end{aligned}
$$

Since the integrand is the algebraic sum of functions belonging to $L(0, \infty)$, the right-hand side of $(9.3)$ is absolutely continuous in $\bar{x}$ for $0 \leqq \bar{x} \leqq 1$, and the limit exists for $\bar{x} \rightarrow 0$, that is,

$$
\left(p^{2}-k^{2}\right) F^{(1)}(p)+2(p-1) F(p)=y(0+) .
$$

According to (7.1) we have then

$$
F(p)=\frac{(p-k)^{-1+1 / k}}{(p+k)^{1+1 / k}}\left[C+y(0+) \int_{p_{0}}^{p}\left(\frac{p+k}{p-k}\right)^{1 / k} d p\right],
$$

where $C$ and $p_{0}, p_{0} \neq \pm k$, are arbitrary finite constants. Since $F(p)$ is unaffected when $k$ is replaced by $-k$, we may assume $k>0$. Clearly

$$
L_{R(p) \rightarrow \infty}\left|\int_{p_{0}}^{p}\left(\frac{z+k}{z-k}\right)^{1 / k} d z\right|=O(|p|) .
$$

The contour avoids a possible branch cut from $-k$ to $k$. By (9.6)

$$
L_{p \rightarrow \infty}|p F(p)|=|y(0+) O(1)| \text {. }
$$

By (a), $F(p)$ is single valued. Hence, by (b), $y(0+)$ vanishes.

* The radial part of the hydrogen wave equation for an $S$ state. 
According to (a), $p=k$ cannot be a branch point. Therefore

$$
k=1 / n, \quad n=1,2, \cdots \text {. }
$$

These are the characteristic values. The associated characteristic functions are given by

$$
y_{n}(x) \doteq C^{\prime}\left[\frac{(1-n p)^{n-1}}{(1+n p)^{n+1}}\right] \doteq C^{\prime \prime}(1-n d / d x)\left(x^{n} e^{-x / n}\right)
$$

The extension of Method 2 to partial differential equations is striking, though not directly obvious. We treat the equation

$$
\begin{aligned}
& L\left(x, \frac{\partial}{\partial x}, \frac{\partial}{\partial t}\right) y(x, t) \equiv(a+x) y_{x x}(x, t)+b y_{t t}(x, t)=r(x, t) \\
& a, b>0 \text {, }
\end{aligned}
$$

with data assigned on the boundaries of the rectangle

$$
0 \leqq x \leqq S, \quad 0 \leqq t \leqq T .
$$

Our procedure yields the formal expansion

$$
\begin{aligned}
y(x, t) & =\sum_{1}^{\infty} Y(n ; x) \sin n \pi t / T ; \\
y(x,-t) & =y(x, 2 T-t)=-y(x, t) .
\end{aligned}
$$

If $(\mathrm{F})$ : the boundary values $y(x, 0), y(x, T)$ vanish, the individual terms in the series of (10.2) satisfy the differential equation. In the contrary case this need no longer be true. The latter situation is easily clarified. We discuss the case that the series of (10.2) is a Fourier series for fixed $x$. Because of the discontinuity of the extended function $y(x, t)(10.3)$ for $t=0, T$, the coefficients $Y(n ; x)$ are at best $O\left(n^{-1}\right)$ * Accordingly, the series of (10.2) cannot be differentiated term by term when $(F)$ is not satisfied.

We deal first with a subrectangle contained between the ordinates $x=0, x=X \leqq S$. We write

$$
F(X ; p, u)=\int_{0}^{T} \int_{0}^{X} e^{-x p+i u t} y(x, t) d x d t .
$$

The operator adjoint to $L(x, \cdots)$ is $\bar{L}(x, \partial / \partial x, \partial / \partial t) \equiv \partial^{2}(a+x) / \partial x^{2}$. $+b \partial^{2} / \partial t^{2}$.. There results from Green's theorem (compare (3.3))

* Courant-Hilbert, Methoden der Mathematischen Physik, 1st edition, p. 53. A similar situation is encountered in the usual Dirichlet problem for the rectangle when the solution is expressed as a double Fourier sine series. 


$$
\begin{aligned}
-M(u, p, \partial / \partial p) F(X ; p, u)+\int_{0}^{T} \int_{0}^{X} e^{-x p+i u t} r(x, t) d x d t \\
=\left.\int_{0}^{T}\left\{(a+x)\left(y_{x}+p y\right)-y\right\} e^{-x p+i u t} d t\right|_{x=0} ^{x=X} \\
+\left.b \int_{a}^{X}\left\{\left(y_{t}-i u y\right) e^{-p x+i u t}\right\} d x\right|_{t=0} ^{t=T} ; \\
M(u, p, \partial / \partial p) .=\partial / \partial p p^{2} .-\left(b u^{2}-a p^{2}\right) .
\end{aligned}
$$

Clearly $G(p, u)=\exp -\left(a p+b u^{2} / p\right)$ satisfies

$$
\begin{aligned}
& \bar{M}(u, p, \partial / \partial p) G(p, u)=\left(-p^{2} \partial / \partial p-\left(b u^{2}-a p^{2}\right)\right) G(p, u)=0 \\
& -\int_{\gamma} G(p, u) M(u, p, \partial / \partial p) F(X ; p, u) d p=\left.p^{2} F(X ; p, u) G(p, u)\right|_{\gamma}
\end{aligned}
$$

The conditions $(\alpha)$ are evidently applicable for finite $u$. The righthand expression in $\left(3.5^{\prime}\right)$ vanishes for (a) $\gamma_{1}$ : the positive real $p$ axis, (b) $\gamma_{2}$ : a circle in the $p$ plane, with center at the origin and unit radius, described in a counter clockwise sense.

We take the special values $u=n \pi / T$ with $n$ an integer and use the imaginary part* of $\left(3.5^{\prime}\right)$ where $M(u, p, \partial / \partial p) F(X ; p, u)$ is replaced by its value according to $\left(3.2^{\prime}\right)$. The inner reason for the success of our procedure is that the $y_{t}$ term (cf. $\left(3.2^{\prime}\right)$ ) drops out. We have

$$
\begin{aligned}
Y_{x}(n, X) V^{\sigma}(n, X)-Y_{x}(n, 0) V^{\sigma} \cdot(n, 0)-Y(n, X) V_{x}^{\sigma}(n, X)=Z^{\sigma}(n, X), & \sigma=1,2, \\
Y(n, X)= & \int_{0}^{T} y(X, t) \sin n \pi t / T d t, \\
V^{\sigma}(n, X)= & (2 \pi i)^{1-\sigma}(a+X) \int_{\gamma_{\sigma}} \exp \left[-(a+X) p-b n^{2} \pi^{2} / p T^{2}\right] d p, \\
Z^{\sigma}(n, X)= & -Y(n, 0) V_{x}^{\sigma}(n, 0) \\
& -b n \pi / T \int_{0}^{X} \frac{V^{\sigma}(n, x)}{a+x}\left(y(x, 0)+(-1)^{n-1} y(x, T)\right) d x \\
& +\int_{0}^{T} \int_{0}^{x} \frac{V^{\sigma}(n, x)}{a+x} \sin \frac{n \pi t}{T} r(x, t) d x d t .
\end{aligned}
$$

The application of the preceding analysis to the complete rectangle of (10.1) yields equations, to be denoted as $\left(1^{\prime \prime}\right)$ and $\left(6^{\prime \prime}\right)$, in which $X$ is replaced by $S$. We may solve $\left(6^{\prime}\right)$ and $\left(6^{\prime \prime}\right)$ formally, by

* The cosine integrals arising from the real part solve the Neumann problem. 
Cramer's rule, for $Y(n, X)$ in terms of integrals of the boundary values and of $r(x, t)$, namely

$$
\begin{gathered}
Y(n, X)=\left|\begin{array}{cccc}
Z^{1}(n, X) & V^{1}(n, X) & 0 & V^{1}(n, 0) \\
Z^{2}(n, X) & V^{2}(n, X) & 0 & V^{2}(n, 0) \\
Z^{1}(n, S)+Y(n, S) V_{x}^{1}(n, S) & 0 & V^{1}(n, S) & V^{1}(n, 0) \\
Z^{2}(n, S)+Y(n, S) V_{x}^{2}(n, S) & 0 & V^{2}(n, S) & V^{2}(n, 0)
\end{array}\right| \\
\div\left|\begin{array}{cc}
V^{1}(n, X) & V_{x}^{1}(n, X) \\
V^{2}(n, X) & V_{x}^{2}(n, X)
\end{array}\right|\left|\begin{array}{cc}
V^{1}(n, 0) & V^{1}(n, S) \\
V^{2}(n, 0) & V^{2}(n, S)
\end{array}\right| .
\end{gathered}
$$

It may be shown that

$$
\begin{aligned}
& V^{1}(n, X)=(2 n \pi / T)(a+X)^{1 / 2} K_{-1}\left((2 n \pi / T)(a+X)^{1 / 2}\right), \\
& V^{2}(n, X)=-(2 n \pi / T)(a+X)^{1 / 2} I_{1}\left((2 n \pi / T)(a+X)^{1 / 2}\right) .
\end{aligned}
$$

The first denominator determinant in (10.4) is the Wronskian of $V^{1}(n, X)$ and $V^{2}(n, X)$ and may be shown to be a nonzero constant. Either from (10.5) or the integral representation, it may be demonstrated that $V^{1}(n, X) / V^{2}(n, X)$ is monotone increasing for $X \geqq 0$, whence it follows that the second denominator determinant in (10.4) cannot be 0 . Thus the solution for $y(n, X)$ is not illusory.

The expansion in (10.2) with coefficients given by (10.4) is an interesting extension of the generalized Schlomilch series, heretofore not treated in the literature. $\dagger$ In case $(\mathrm{F})$, if the data go to 0 at $t=0$ or $T$ and are, for instance, of class $C^{2}$ and $r(x, t)=0$, it is easy to establish the convergence of the series in (10.2) to a solution. However we defer the investigation of the properties of such expansions. $\ddagger$

UNIVERSITY OF ILLINOIS AND PuRdue University

* G. N. Watson, Theory of Bessel Functions, pp. 80, 181, 183.

$\dagger$ Watson, ibid., p. 622.

$\ddagger$ The introduction of an accessory line $x=X$ and subsequent elimination of derivatives by taking two regions is central in all applications of the second method to the hyperbolic and parabolic equations also. For mixed problems and a finite range on either variable the corresponding parameter is given discrete values; for the Cauchy problem both $u$ and $p$ are continuous. Method 1 is also available. 